\title{
The Female Mosquito's Quest for Blood: Implications for Disease Cycles ${ }^{1}$
}

\author{
Stephanie L. Richards, Sheri L. Anderson, and Chelsea T. Smartt ${ }^{2}$
}

The familiar buzzing in your ear signals that a blood thirsty female mosquito has found you. It is not personal, as she is simply following her biological instinct to survive and produce offspring. You might not mind donating a couple of microliters of your blood, but her saliva will leave an itchy welt and she might leave you with a debilitating or deadly disease. This is the story of the female mosquito's quest for blood and how it may affect our lives.

\section{Why do only female mosquitoes feed on blood?}

Both male and female mosquitoes have a proboscis that is used to suck water for hydration and plant nectar for energy. When mosquitoes feed, their mouthparts secrete saliva, allowing them to also ingest crystallized sugar sources. However, female mosquito mouthparts are slightly different than male mosquito mouthparts because the female is able to penetrate animal tissues to feed. The female mosquito is the only sex that feeds on vertebrate blood and can ingest approximately two times her weight (Figure 1). The protein from the blood is used primarily for the development of her eggs, but it can also be used as an energy source. There are a limited number of mosquito species that do not require blood for egg development, and they can create a batch of eggs from the sugar derived from plant nectar. A female mosquito can lay several batches of eggs during her lifetime, depending on environmental factors affecting her survival rate and availability of blood sources.

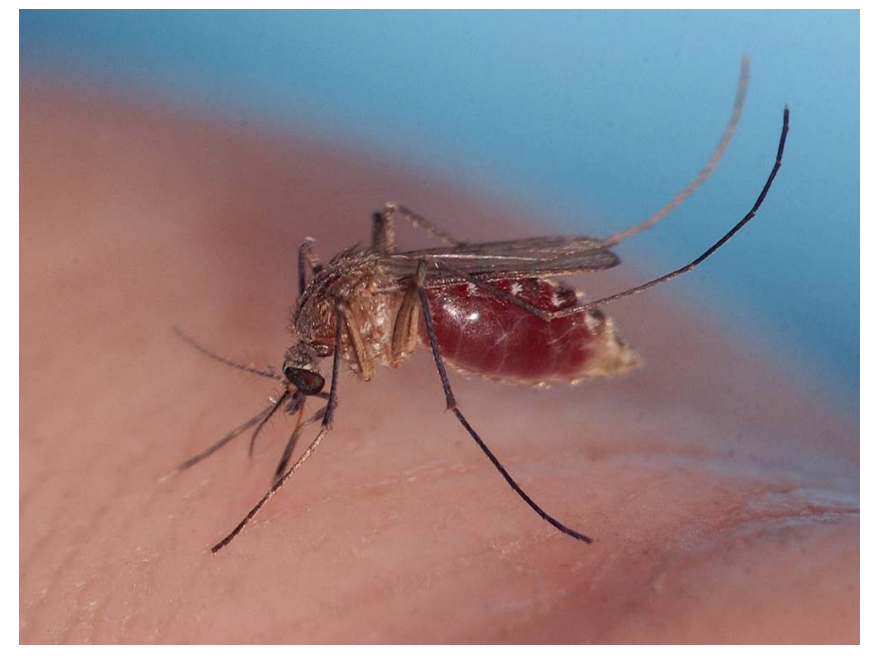

Figure 1. Female Culex pipiens quinquefasciatus taking a blood meal from a human. Credits: Jim Newman, UF/IFAS

1. This document is ENY-855 (IN811), one of a series of the Entomology and Nematology Department, Florida Cooperative Extension Service, Institute of Food and Agricultural Sciences, University of Florida. First printed: October 2009. Please visit the EDIS Website at http://edis.ifas.ufl.edu.

2. Stephanie L. Richards, research assistant scientist, Sheri L. Anderson, graduate student, and Chelsea T. Smartt, assistant professor, Florida Medical Entomology Laboratory, University of Florida, IFAS, 200 9th Street Southeast, Vero Beach, FL 32962. 


\section{What makes a blood source suitable to a female mosquito?}

Different mosquito species have evolved to blood feed on various types of vertebrate hosts. For instance, some mosquitoes prefer mammalian blood, while others would rather feed solely on birds. There are even mosquitoes specialized to feed on amphibians or reptiles. Opportunistic feeding mosquitoes will feed on any animal, depending on host availability during the host-seeking period. For instance, some mosquito species search for hosts during daylight hours while others are active at dawn and dusk. The attractiveness of a blood meal host and the subsequent feeding success of a mosquito depend on characteristics such as host size, proximity to mosquito habitats, host abundance during mosquito host-seeking periods, and host defense mechanisms.

\section{Why are some people more attractive to mosquitoes than others?}

There can also be differences in host attractiveness for those mosquito species that feed on humans, and it is not uncommon for some people to be more attractive to mosquitoes than others. Several studies have shown that heat, carbon dioxide, moisture, and host odors attract female mosquitoes and characteristics of these compounds vary between people. Scientists are currently studying the reasons for the difference in attractiveness in order to assist development of repellent compounds for preventing blood feeding and attractants for mosquito traps.

\section{Why is knowledge of a mosquito's blood meal host preference important to understanding how diseases are spread?}

Mosquitoes are the primary method of arthropod-borne pathogen transmission between animals. Urban and suburban sprawl continues to facilitate interactions between humans and mosquitoes. In addition to variation in a mosquito's blood meal host preference, there are differences in vector competence (i.e. the ability of a mosquito to become infected with and subsequently transmit a pathogen). Knowledge of the interplay between a mosquito's host feeding pattern and vector competence is an essential component in determining how diseases are spread because control measures can be targeted to specific mosquito species and populations.

Several pathogens of public health importance in the United States such as Eastern equine encephalitis virus (EEEV), St. Louis encephalitis virus (SLEV), Western equine encephalitis virus (WEEV), and West Nile virus (WNV) are maintained in enzootic cycles between birds and bird-feeding mosquitoes in the genera Culex and Culiseta (Figure 2). Humans and other mammals are affected by these viruses when Culex and other genera of mosquitoes feed on birds and then mammals, acting as bridge vectors and spreading the virus to additional hosts.

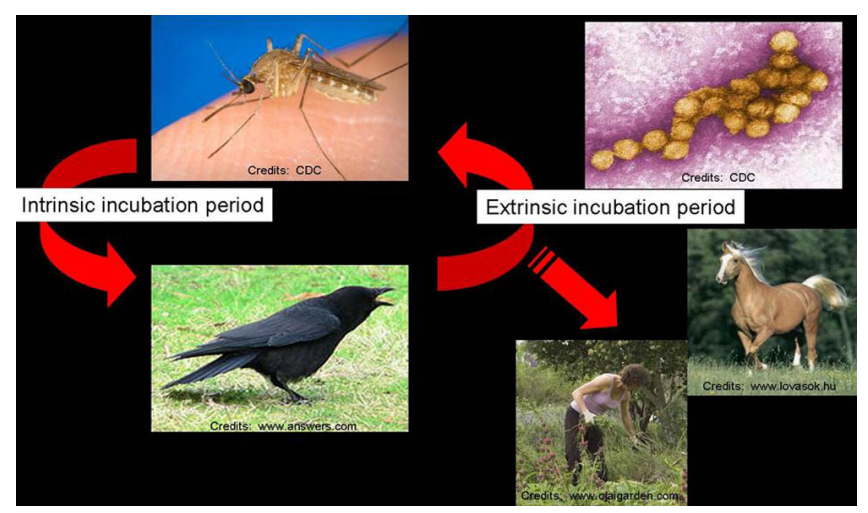

Figure 2. Virus transmission cycle Credits:

Everglades virus (EVEV) is another arthropod-borne pathogen that is primarily maintained in an enzootic cycle between Culex species and rodents, with incidental human epidemics. The EVEV in Florida is a subtype of the more virulent Venezuelan equine encephalitis virus (VEEV) found in Central and South America.

Although malaria has not been a public health issue in the United States since the 1950s, global travel of infected people has lead to isolated disease incidences in several states. There have been three different focal outbreaks of malaria in Florida since the 1990s, likely propagated by local populations of mosquitoes (http://edis.ifas.ufl.edu/MG103). The protozoan malaria parasite is cycled between people and mosquitoes of the genus Anopheles.

Dengue virus (DENV) and chikungunya virus (CHIKV) are maintained in epidemic cycles between humans and mosquitoes in the Aedes genus (Figure $3)$. The last large dengue epidemic in the continental 
United States occurred in Florida in the 1930s. However, several dengue cases have been imported into Florida since the 1990s due to infected travelers returning from regions where dengue is endemic. Furthermore, a dengue outbreak occurred in Hawaii from 2001-2002 and Puerto Rico has experienced several epidemics over the last 20 years. Chikungunya virus cases have also occurred in the United States due to infected travelers returning from other countries where chikungunya outbreaks are occurring. Because Florida is home to the primary mosquito vectors of these two viruses, it is possible that DENV and CHIKV will emerge or re-emerge in the United States.

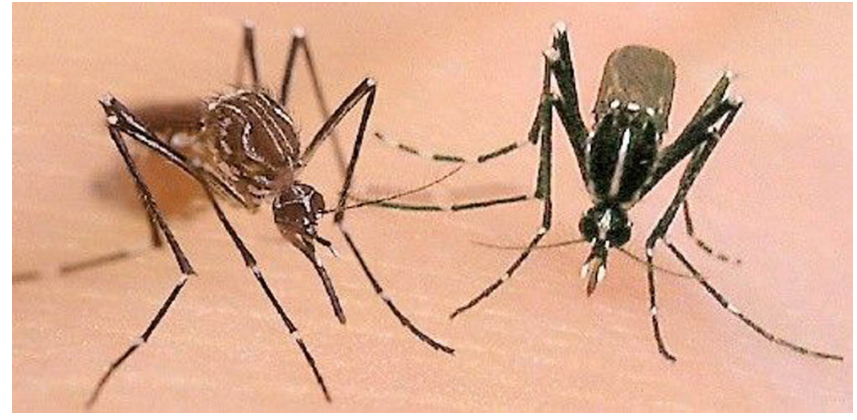

Figure 3. Aedes aegypti and Aedes albopictus. Credits: Jim Newman, UF/IFAS

\section{Host preference of selected Florida mosquitoes and relevance to endemic and emerging arthropod-borne diseases}

There have been 80 mosquito species identified in Florida to date. Several of these species have been incriminated in arthropod-borne disease cycles in Florida. Culiseta melanura feeds primarily on birds and plays a major role in maintaining enzootic cycles of EEEV, SLEV, and WNV. Coquillettidia perturbans feeds on both birds and mammals and is also involved in the EEEV transmission cycle. Culex nigripalpus, $C x$. restuans, $C x$. salinarius, and $C x$. pipiens quinquefasciatus feed on both birds and mammals and likely contribute to enzootic cycles in wild animal populations and epidemic cycles in human populations of EEEV, SLEV, and WNV. Culex cedecei prefers to feed on rodents and is the primary vector of EVEV in Florida.
Aedes albopictus and Ae. aegypti feed primarily on mammals and are daytime host-seekers so are primarily nuisance species. However, these Aedes species are the primary vectors of CHIKV and DENV in other parts of the world and could play a major role if these viruses emerge in Florida. Aedes taeniorhynchus (Figure 4) and Ae. sollicitans are salt-marsh mosquitoes that are a major nuisance, but have not been implicated as virus vectors because of their limited vector competence for Florida arboviruses. However, these two species are good vectors of VEEV and could be implicated in transmission cycles if this more virulent form of EVEV were to emerge in Florida.

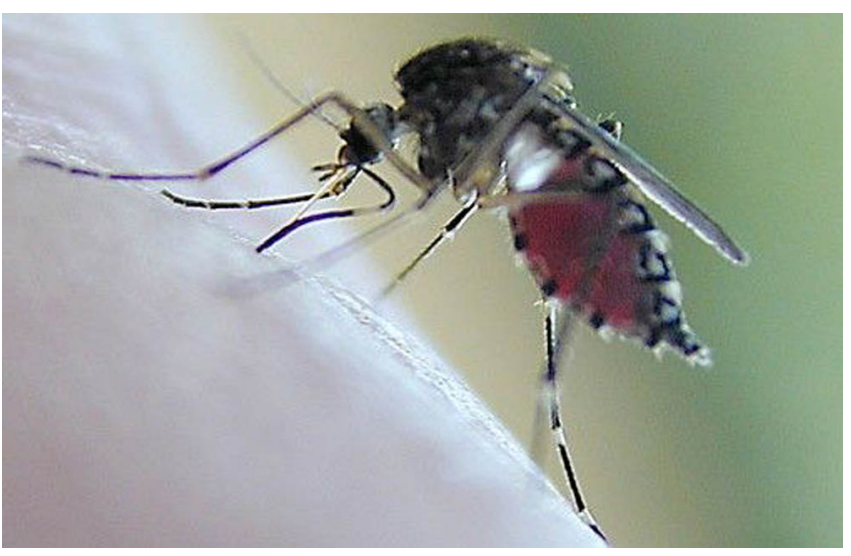

Figure 4. Female Aedes taeniorhynchus taking a blood meal from a human. Credits: Jim Newman, UF/IFAS

Anopheles quadrimaculatus and An. crucians feed primarily on humans and other large mammals in Florida. In countries where malaria is prevalent, Anopheles mosquitoes play important roles in the propagation of this disease. These Florida mosquito species may be involved in transmission cycles of the protozoan malaria parasite if this disease ever re-emerges in the United States.

Florida mosquito species in the genera Mansonia, Orthopodomyia, Psorophora, Toxorhynchites, Uranotaenia, and Wyeomyia are not known to transmit pathogens that are currently found in Florida so are not of public health importance at this time. However, due to the increased risk of pathogen importation, mosquitoes from these genera could be implicated in emerging disease cycles as some are involved in disease cycles in other regions of the world. 
More information on Florida mosquitoes can be found at the website of the Florida Medical

Entomology Laboratory (http://fmel.ifas.ufl.edu/). 